\title{
ON SIMULATIONS ABOUT THE PRECISION OF NON UNIFORM HYBRID FILTER BANK ANALOG/DIGITAL CONVERTERS
}

\author{
Jean-Luc Collette and Michel Barret
}

\author{
SUPELEC \\ Signal Processing Systems Team \\ 2, rue E. Belin, 57070 Metz (France) \\ \{Jean-Luc.Collette, Michel.Barret\}@ supelec.fr
}

\begin{abstract}
In this paper we present a theoretical study of the errors due to imperfect reconstruction and to quantization in hybrid filter bank (HFB) analog/digital (A/D) converters, that are non uniform i.e., with $K$ channels and an arbitrary ratio $M$ of the $M$-fold expanders $(M \leq K)$. We propose then a new method of simulation for such HFB A/D converters that avoids any numerical computation of differential equation solutions. The results of simulation are compared with theoretical ones. Moreover, in HFB A/D converter studies, the effect of quantization noise is generally indicated by its average power at the output of the HFB, when it is $M$-cyclostationary. This paper shows on an example that the variance of the global error at the HFB output can vary a lot in a period of $M$ samples.
\end{abstract}

\section{INTRODUCTION}

Hybrid filter bank (HFB) Analog/Digital (A/D) systems have been studied for several years ([8], [4], [5], [2]). The motivation of this research comes from the demand for higher data rates encountered in many domains, as in wireless communication. In [1], a new HFB having more degrees of freedom than the classical ones has been studied, it is represented in Fig. 1. In this quoted paper, a natural generalization of the

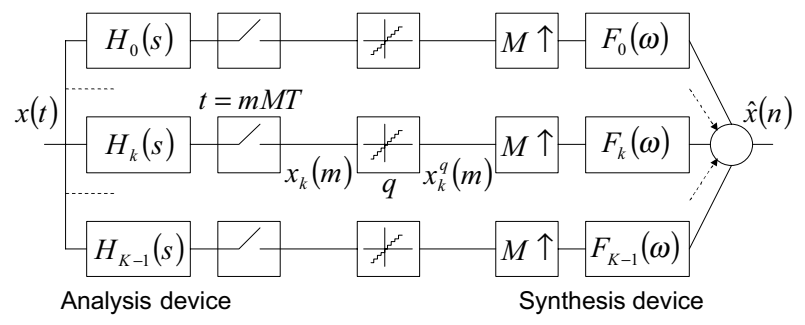

Fig. 1. HFB A/D converter with $K$ channels. In each channel there is, from left to right, an analog analysis filter, a $M T$ periodic sampler, an infinite uniform scalar quantizer, a $M$ fold expander and a digital synthesis filter.

classical HFB was presented, called non uniform HFB and obtained by reducing the ratio $M$ of the $M$-fold expanders without modifying the number $K$ of channels. It was then shown how the excess parameters can be used for minimizing the aliasing and the distorsion under the constraint of a given quantization noise amplification $G$, as it was done in [3]. Consequently, constrained minimization was applied in such a way that the output signal $\hat{x}(n)$ is as close to the ideal sampled signal $x^{\star}(n)=x(n T)$ as possible, where $x(t)$ is the input signal. It is well known [6] that in each channel, the quantization noise obtained after the synthesis filter is $M$ cyclostationary. In [1], the mean aliasing which was calculated according to the constraint $G$, gave only qualitative information about the precision of the HFB A/D converter. In this paper, all the values of the periodic- $M$ variance of the error signal $x^{\star}(n)-\hat{x}(n)$ are calculated as figures of merit.

The design of the HFB A/D according to the method described in [1] gives the impulse response coefficients of the $K$ digital synthesis FIR filters $f_{k}(n)(-N \leq n<N$ and $0 \leq k<K)$. This design uses the $2 \pi$-periodic functions $\left(H_{k}^{d}(\omega)\right)_{0 \leq k<K}$ which verify

$$
\forall \omega \in]-\pi, \pi], \quad H_{k}^{d}(\omega)=H_{k}(j \omega / T),
$$

where $H_{k}(j \Omega)(0 \leq k<K)$ are the frequency responses of the analog analysis filters. The input signal $x(t)$ is supposed to be a wide sense stationary (WSS) stochastic process with zero mean. The sampling Nyquist condition-or possibly stronger-on the input signal $x(t)$ is assumed:

$$
\left.\exists \omega_{\max } \in\right] 0 ; \pi\left[, \forall \Omega \in \mathbb{R},|T \Omega| \geq \omega_{\max } \Rightarrow S_{x}(\Omega) \approx 0\right.
$$

with $S_{x}(\Omega)$ the power spectrum density (psd) of $x(t)$.

In the next section, the variance of the error $x^{\star}(n)-\hat{x}(n)$ is given without considering the quantization. In Section 3, the quantization noise is considered. In Section 4, a new simulation method for HFB A/D converters is described. The proposed simulation has the advantage over classical ones because it does not require any numerical solution of differential equations. The theoretical study of sections 2 and 3 permits to evaluate independently the error due to imperfect recon- 
struction from the error due to quantization. And so the theoretical and simulated global errors can be compared. Finally, practical results are presented in section 5 in order to validate theoretical results.

\section{HFB WITHOUT QUANTIZATION}

In Fig. 1, the entire HFB A/D converter is described. The signals $x_{k}(m)(m \in \mathbb{Z}$ and $0 \leq k<K)$ are the $K$ outputs of the analysis device. When no quantization is considered and the input signal $x(t)$ is applied (i.e., when $x_{k}^{q}(m)=x_{k}(m)$ for $0 \leq k<K$ and $m \in \mathbb{Z}$ ), let $\hat{x}^{0}(n)$ denote the HFB output signal. It can be expressed according to the ideal signal $x^{\star}(n)$, the coefficients of the digital synthesis filters $f_{k}(n)$ and coefficients $h_{k}^{d}(n)$ which are the inverse Fourier transforms of the functions $H_{k}^{d}(\omega)$ introduced in (1):

$$
\begin{aligned}
\hat{x}^{0}(n) & =\sum_{k=0}^{K-1} \sum_{\ell=-\infty}^{+\infty} f_{k}(n-M \ell) x_{k}(\ell) \\
& =\sum_{k=0}^{K-1} \sum_{\ell=-\infty}^{+\infty} f_{k}(n-M \ell) \sum_{m=-\infty}^{+\infty} h_{k}^{d}(m) x^{\star}(M \ell-m) .
\end{aligned}
$$

Note that the summation on $\ell$ is actually finite, since the synthesis filters are FIR. Since the input signal $x(t)$ is supposed WSS and centered, the sampled signal $x^{\star}(n)$ is a centered discret time WSS stochastic process, with an autocovariance function noted $\phi_{x}(n)$. The output signal $\hat{x}^{0}(n)$ is also centered and the variance $\varepsilon^{2}(n)$ of the error $x^{\star}(n)-\hat{x}^{0}(n)$ can be expressed as

$$
\varepsilon^{2}(n)=\phi_{x}(0)-A+B
$$

with

$$
\begin{gathered}
A=2 \sum_{k=0}^{K-1} \sum_{l} f_{k}(n-M l) \sum_{m} h_{k}^{d}(m) \phi_{x}(n-M l+m) \\
B=\sum_{k, k^{\prime}} \sum_{l, l^{\prime}} f_{k}(n-M l) f_{k^{\prime}}\left(n-M l^{\prime}\right) \sum_{m, m^{\prime}} h_{k}^{d}(m) h_{k^{\prime}}^{d}\left(m^{\prime}\right) \times \\
\phi_{x}\left(M l-m-M l^{\prime}+m^{\prime}\right)
\end{gathered}
$$

Note that the summation on the indexes $m$ and $m^{\prime}$ is infinite. In order to get rid of this problem, the equation (6) can be rewritten by using the power spectrum density of $x^{\star}(n)$, noted $S_{x}(\omega)$, and the functions $H_{k}^{d}(\omega)$ of equation (1):

$$
\begin{aligned}
A= & \frac{1}{\pi} \sum_{k=0}^{K-1} \sum_{l} f_{k}(n-M l) \times \\
& \int_{-\pi}^{\pi} \overline{H_{k}^{d}(\omega)} S_{x}(\omega) e^{i \omega(n-M l)} d \omega \\
B= & \frac{1}{2 \pi} \sum_{k, k^{\prime}=0}^{K-1} \sum_{l, l^{\prime}} f_{k}(n-M l) f_{k^{\prime}}\left(n-M l^{\prime}\right) \times \\
& \int_{-\pi}^{\pi} H_{k}^{d}(\omega) \overline{H_{k^{\prime}}^{d}(\omega)} S_{x}(\omega) e^{i \omega M\left(l-l^{\prime}\right)} d \omega
\end{aligned}
$$

This expression shows that the signal $x^{\star}(n)-\hat{x}^{0}(n)$ is $M$ cyclostationary and its variance can be expressed with a finite summation on the indexes $l$ and $l^{\prime}$ in (8).

\section{HFB WITH QUANTIZATION}

Conditions of high resolution quantization are assumed, that is the $K$ quantization noises are mutually wide sense stationary, white, centered and uncorrelated. Let $b_{k}(m)=x_{k}(m)-$ $x_{k}^{q}(m)$ be the quantization noise of the $k^{\text {th }}$ channel and $\sigma_{k}^{2}$ its variance. Let $v_{k}(n)$ be the output of the filter $F_{k}(\omega)$ when $x_{k}^{q}(m)=b_{k}(m)$ in Fig. 1 and let $v(n)=\sum_{k=0}^{K-1} v_{k}(n)$ be the quantization noise at the output of the HFB; $v(n)=$ $\hat{x}(n)-\hat{x}^{0}(n)$ where $\hat{x}^{0}(n)$ is the output signal without quantization in the HFB (see Section 2). Let us introduce the polyphase representation of filter $F_{k}(\omega)$ :

$$
F_{k}(\omega)=\sum_{\ell=0}^{M-1} F_{k, \ell}(M \omega) e^{-i \ell \omega} \quad(0 \leq k<K),
$$

and the matrix $\mathbf{F}(\omega)=\left[F_{k, \ell}(\omega)\right](0 \leq \ell<M, 0 \leq k<K)$ of dimension $M \times K$, which is the polyphase representation of the synthesis filter bank. The $k^{\text {th }}$ column, $\mathbf{F}_{k}(\omega)$, of $\mathbf{F}(\omega)$ is the $M$-fold polyphase representation of the filter $F_{k}(\omega)$. The $M$-fold blocked versions $\mathbf{v}_{k}(m)=\left(v_{k}(m M), v_{k}(m M-\right.$ $\left.1), \ldots, v_{k}(m M-M+1)\right)^{T}$ of $v_{k}(0 \leq k<K)$ are mutually WSS ([6]) and uncorrelated. For a matrix $\mathbf{A}, \mathbf{A}^{T}$ (resp. $\mathbf{A}^{H}$ ) denotes the transpose (resp. the adjoint) of $\mathbf{A}$. The power spectra (psd matrix $\mathbf{S}_{\mathbf{v}_{k}}(\omega)$ ) of $\mathbf{v}_{k}$ is given by

$$
\mathbf{S}_{\mathbf{v}_{k}}(\omega)=\sigma_{k}^{2} \mathbf{F}_{k}(\omega) \mathbf{F}_{k}(\omega)^{H}
$$

hence the psd matrix $\mathbf{S}_{\mathbf{v}}(\omega)$ of $\mathbf{v}$ ( $\mathbf{v}$ is the $M$-fold blocked version of $v(n))$ satisfies

$$
\mathbf{S}_{\mathbf{v}}(\omega)=\sum_{k=0}^{K-1} \sigma_{k}^{2} \mathbf{F}_{k}(\omega) \mathbf{F}_{k}(\omega)^{H}=\mathbf{F}(\omega) \boldsymbol{\Delta} \mathbf{F}(\omega)^{H}
$$

where $\Delta$ is the variance-covariance matrix of the column vector $\underline{b}(m)=\left(b_{0}(m), \ldots, b_{K-1}(m)\right)^{T}$. Hence the average power $\mathrm{E}\left[|v(n)|^{2}\right]$ of the quantization noise at the HFB output is periodic- $M$ with, for $0 \leq \ell<M$,

$$
\begin{aligned}
\mathrm{E}\left[v(m M-\ell)^{2}\right] & =\sum_{k=0}^{K-1} \frac{\sigma_{k}^{2}}{2 \pi} \int_{-\pi}^{+\pi}\left|F_{k, \ell}(\omega)\right|^{2} d \omega \\
& =\sum_{k=0}^{K-1} \sigma_{k}^{2} \sum_{u} f_{k}(M u-\ell)^{2}
\end{aligned}
$$

and its temporal average $P_{a v}=\frac{1}{M} \sum_{\ell=0}^{M-1} \mathrm{E}\left[v(m M-\ell)^{2}\right]$ is given by the relation

$$
P_{a v}=\frac{1}{2 \pi M} \sum_{k=0}^{K-1} \sigma_{k}^{2} \int_{-\pi}^{+\pi}\left|F_{k}(\omega)\right|^{2} d \omega=\frac{1}{M} \sum_{k=0}^{K-1} \sigma_{k}^{2} \sum_{u} f_{k}(u)^{2}
$$


So the variance of the global error can be expressed, assuming that the error due to unperfect reconstruction is uncorrelated with the error due to quantization:

$$
\mathrm{E}\left[\left|x^{\star}(n)-\hat{x}(n)\right|^{2}\right]=\varepsilon^{2}(n)+\mathrm{E}\left[v(n)^{2}\right] .
$$

\section{SIMULATION METHOD}

In our simulations it is assumed that each FIR synthesis filter $F_{k}(\omega)$ has $2 N$ non zero coefficients. In order to preserve the symmetry between the $K$ quantizers (same quantization step and same number of bits) with an optimal bit allocation [7], we adjust the gain of each analog filter (see Fig. 2) to equalize the variance of the subband signals $x_{k}(m)(0 \leq k<K)$ when the input signal has a constant psd in the useful frequency band (see equation (2)). Hence all the quantizer have the same distortion $\sigma^{2}$, and the value

$$
G=\frac{1}{M} \sum_{k=0}^{K-1} \sum_{n=-N}^{N-1} f_{k}^{2}(n)
$$

represents the noise amplification gain. We used then the method presented in [1] for computing the optimal synthesis filters of the HFB.

Classical numerical methods of simulation for analog devices lead to an approximation error, due to the numerical resolution of the differential equations of filtering. This error could be mixed with the global error (14) so that the interpretation of practical results would be wrong. In order to get rid of this problem, an input signal

$$
x(t)=\sum_{p=1}^{P} \sqrt{2} A_{p} \sin \left(\Omega_{p} t+\Phi_{p}\right)
$$

can be considered, where $\left(\Phi_{p}\right)_{1 \leq p \leq P}$ are $P$ independent random variables uniformly distributed in the interval $[0 ; 2 \pi]$. The frequencies $\left\{\Omega_{p}\right\}_{1 \leq p \leq P}$ are determinist and regularly spaced in the interval $\left[0 ; \omega_{\max } / T\right]$. The frequency $\omega_{\max }$ introduced in (2) represents the sampling condition (it is less than $\pi$ when this condition is stronger than that of Nyquist). So the ideal signal in this case is

$$
x^{\star}(n)=\sum_{p=1}^{P} \sqrt{2} A_{p} \sin \left(\Omega_{p} T n+\Phi_{p}\right)
$$

its correlation function satisfies $\phi_{x}(k)=\sum_{p=1}^{P} A_{p}^{2} \cos \left(\Omega_{p} T k\right)$ and its power spectrum density is given by

$$
S_{x}(\omega)=\pi \sum_{p=1}^{P} A_{p}^{2}\left[\delta\left(\omega-\Omega_{p} T\right)+\delta\left(\omega+\Omega_{p} T\right)\right]
$$

for $\omega \in]-\pi ; \pi[$. For this particular power spectrum density, the integrals in (7) and (8) can be replaced by finite summations. In a steady state, the $K$ output signals of the HFB analysis device are:

$$
\begin{aligned}
& x_{k}(m)= \sum_{\substack{p=1\\
}}^{P}\left|H_{k}\left(i \Omega_{p}\right)\right| \sqrt{2} A_{p} \times \\
& \sin \left[\Omega_{p} T M m+\Phi_{p}+\arg \left(H_{k}\left(i \Omega_{p}\right)\right)\right]
\end{aligned}
$$

It is then easy to compute the output signal, assuming that the unsteady state vanished:

$$
\hat{x}(n)=\sum_{k=0}^{K-1} \sum_{m} x_{k}^{q}(m) f_{k}(n-M m) .
$$

For a given design of a HFB A/D converter, simulations can be done with the method described in this section. Assuming that the power spectrum density of the ideal signal $x^{\star}(n)$ is given by equation (18), the variance of the global error (14) can be estimated and this estimation can be compared with the theoretical value obtained from (5) - with (78) - and (12) —with $\sigma_{k}^{2}=\sigma^{2}=q^{2} / 12$, where $q$ is the quantization step shared by all the quantizers.

\section{PRACTICAL RESULTS}

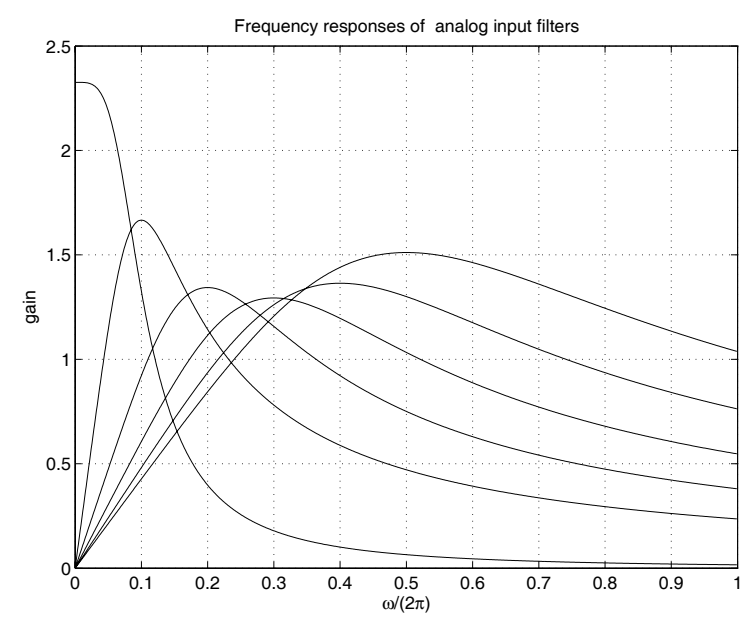

Fig. 2. Frequency response magnitudes of the analog analysis filters.

In order to do the estimation, $N_{\text {iter }}$ realizations of the random signal $x^{\star}(n)$ (see section 4) are considered. For each realization, the output signal $\hat{x}(n)$ is calculated, according to equation (20) and the empirical mean square of $x^{\star}(n)-\hat{x}(n)$ is calculated for each time $n$ with the $N_{\text {iter }}$ realizations. A given design of HFB is considered with $K=6$ (number of channels) and $M=4$ (ratio of the $M$-fold expander). The 6 digital synthesis filters have 32 coefficients $(N=16)$. A 
sampling condition stronger than that of Nyquist is assumed with $\omega_{\max }=\pi-\frac{\pi}{20}$. The 6 analog analysis filters are second order filters, as shown in Fig. 2. This design corresponds to $G=6.81 \mathrm{~dB}$, a mean aliasing equal to $-100.2 \mathrm{~dB}$ and aliasing functions represented in Fig. 3 .

In equation (18), the amplitude values are $A_{p}=1 / \sqrt{P}$ for $1 \leq p \leq P$, in order to have $\phi_{x}(0)=1$. We choose $P=$ 400 and $N_{\text {iter }}=200$. For a given value of the quantization step, the right member of equation (14) is evaluated thanks to equation (5) - with (7-8) - and equation (12). The results are displayed on Table 1 according to the time $n$ modulo $M$. Then a simulation is done, following the method described in section 4 and the estimation of the variance is obtained as it is described at the end of the previous section. The results are displayed on Table 1.

\begin{tabular}{|l|c|c|c|c||c|c|c|c|}
\hline & \multicolumn{6}{|c|}{$\operatorname{Var}\left[x^{\star}(n)-\hat{x}(n)\right]$ in dB } \\
\hline & \multicolumn{5}{|c|}{ theoretical } & \multicolumn{5}{c|}{ estimated } \\
\hline$n \bmod M$ & 0 & 1 & 2 & 3 & 0 & 1 & 2 & 3 \\
\hline$q=2.10^{-4}$ & -80 & -76 & -75 & -82 & -79 & -76 & -75 & -82 \\
\hline$q=10^{-4}$ & -85 & -82 & -80 & -88 & -85 & -82 & -81 & -88 \\
\hline$q=5.10^{-5}$ & -88 & -86 & -84 & -91 & -88 & -86 & -85 & -91 \\
\hline$q=2.10^{-5}$ & -89 & -89 & -86 & -93 & -90 & -89 & -87 & -94 \\
\hline$q=10^{-5}$ & -90 & -89 & -87 & -94 & -91 & -90 & -87 & -94 \\
\hline
\end{tabular}

Table 1. Theoretical and estimated values of the periodic- $M$ variance of the global error $x^{\star}(n)-\hat{x}(n)$ for the different values of $n$ modulo $M$.

We can see on Table 1, comparing estimated with theoretical values that the hypothesis assumed in order to obtain the theoretical results in sections 2 and 3 are relevant. If the quantization step becomes too small, the performances do not increase any more. Moreover this study highlights the fact that the average variance of the global error is not a sufficient information for evaluating the performances of an HFB A/D converter. Indeed, a variation of nearly $7 \mathrm{~dB}$ can be observed on a $M$-period of the variance.

\section{CONCLUSION}

In order to give a relevant information on the precision of a non uniform HFB A/D converter with $M$-fold expanders, we gave the expression of the periodic- $M$ variance of the global error. This theoretic study has been validated by a simulation method, which get rid of problems related to the numerical computation of differential equations due to analog devices. We shown on an example that the variance of the global error can vary a lot in a period of $M$ samples.

\section{REFERENCES}

[1] J.-L. Collette, M. Barret, P. Duhamel and J. Oksman, "On hybrid filter bank A/D converters with arbitrary
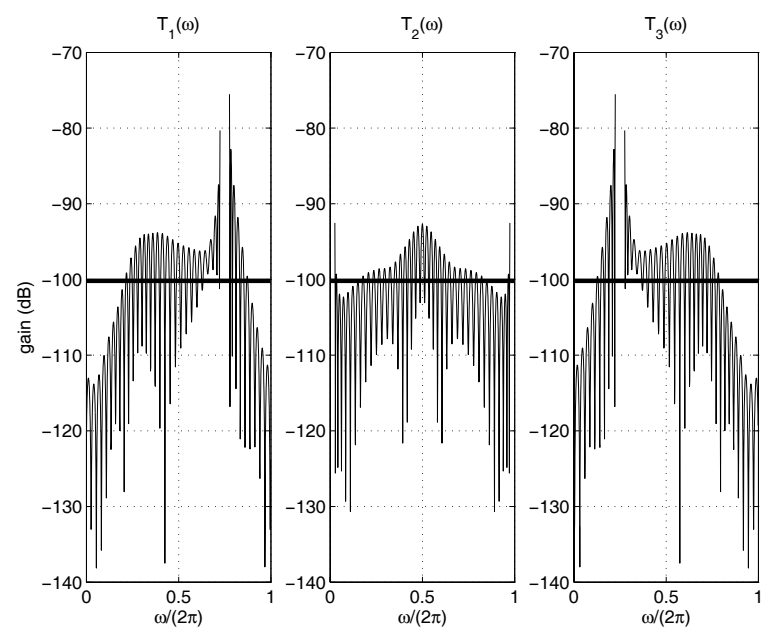

Fig. 3. Aliasing functions (the bold line corresponds to the mean aliasing).

over-sampling rate", Proc. 4th IEEE Int. Symp. on Image and Signal Processing and Analysis, pp. 157-160, Zagreb (Croatia), Sep. 2005.

[2] J.-L. Collette, M. Barret and J. Oksman, "Synthesis of hybrid filter banks for A/D conversion: a frequency domain approach", Proc. EUSIPCO, pp. 109-112, Vienna (Austria), Sep. 2004.

[3] K. Gosse, F. Moreau de Saint-Martin and P. Duhamel, "Filter bank design for minimum distortion in presence of subband quantization", Proc. ICASSP, Atlanta (USA), May 1996.

[4] P. Löwenborg, H. Johansson and L. Wanhammar, "A class of two-channel approximately perfect reconstruction hybrid analog/digital filter banks", Proc. IEEE Int. Symp. Circ. Syst., Geneva, May 2000.

[5] T. Petrescu, C. Lelandais-Perrault and J. Oksman, "Synthesis of hybrid filter banks for A/D conversion with implementation constraints - mixed distortion/aliasing optimization”, Proc. ICASSP, Montreal, May 2004.

[6] V. Sathe and P. P. Vaidyanathan, "Effects of multirate systems on the statistical properties of random signals," IEEE Trans. on Signal Processing, vol. ASSP-41, pp. 131-146, Jan 1993.

[7] P. P. Vaidyanathan, Multirate Systems and Filter Banks, Englewood Cliffs, NJ: Prentice-Hall, 1993.

[8] S. R. Velasquez, T. Q. Nguyen, S. R. Broadstone, "Design for hybrid filter banks for analog/digital conversion", IEEE Trans. Sig. Proc., vol. 46, no. 4, pp. 956967, Apr. 1998. 